\title{
A note on wave equation in Einstein and de Sitter space-time
}

\author{
Anahit Galstian, ${ }^{1, a)}$ Tamotu Kinoshita, ${ }^{2, b)}$ and Karen Yagdjian ${ }^{1, c)}$ \\ ${ }^{1}$ Department of Mathematics, University of Texas-Pan American, 1201 W. University \\ Drive, Edinburg, Texas 78541-2999, USA \\ ${ }^{2}$ Institute of Mathematics, University of Tsukuba, Tsukuba, Ibaraki 305-8571, Japan
}

(Received 17 August 2009; accepted 22 March 2010; published online 13 May 2010)

\begin{abstract}
We consider the wave propagating in the Einstein and de Sitter space-time. The covariant d'Alembert's operator in the Einstein and de Sitter space-time belongs to the family of the non-Fuchsian partial differential operators. We introduce the initial value problem for this equation and give the explicit representation formulas for the solutions. We also show the $L^{p}-L^{q}$ estimates for solutions. (c) 2010 American Institute of Physics. [doi:10.1063/1.3387249]
\end{abstract}

\section{INTRODUCTION}

The current note is concerned with the wave propagating in the universe modeled by the cosmological models with expansion. We are motivated by the significant importance of the solutions of the partial differential equations arising in the cosmological problems for our understanding of the universe. While there exists extensive literature on the hyperbolic equations, the question of initial value problems for the wave equation in the curved spaces with singularities, and, in particular, in the Einstein and de Sitter space-time, which are well posed and preserve many features of the classical waves, remains unresolved.

The homogeneous and isotropic cosmological models possess highest symmetry, which makes them more amenable to rigorous study. Among them, Friedmann-Lemaître-Robertson-Walker models are mentioned. The simplest class of cosmological models can be obtained if we assume that the metric of the slices of constant time is flat and that the space-time metric can be written in the form

$$
d s^{2}=-d t^{2}+a^{2}(t)\left(d x^{2}+d y^{2}+d z^{2}\right),
$$

with an appropriate scale factor $a(t)$. (See, e.g. Ref. 22.) The assumption that the universe is expanding leads to the positivity of the time derivative $(d / d t) a(t)$. The time dependence of the function $a(t)$ is determined by the Einstein field equations for gravity,

$$
R_{\mu \nu}-\frac{1}{2} g_{\mu \nu} R=-8 \pi G T_{\mu \nu} .
$$

The metric of the Einstein and de Sitter universe (EdeS universe) is a particular member of the Friedmann-Robertson-Walker metrics,

$$
d s^{2}=-d t^{2}+a^{2}(t)\left[\frac{d r^{2}}{1-K r^{2}}+r^{2} d \Omega^{2}\right]
$$

where $K=-1,0$, or +1 , for a hyperbolic, flat, or spherical spatial geometry, respectively. The Einstein equations are simply

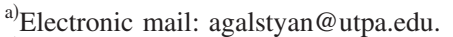

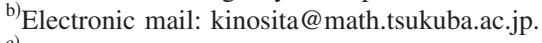

${ }^{c)}$ Electronic mail: yagdjian@utpa.edu.
} 


$$
\dot{\rho}=-3(\rho+p) \frac{\dot{a}}{a}, \quad \frac{\ddot{a}}{a}=-\frac{4 \pi}{3}(\rho+3 p), \quad\left(\frac{\dot{a}}{a}\right)^{2}=\frac{8 \pi}{3} \rho-\frac{K}{a^{2}},
$$

where $\rho$ is the proper energy density and $p$ is pressure. For pressureless matter distributions ( $p$ $=0)$ and vanishing spatial curvature $(K=0)$ in the EdeS universe the solution to the Eqs. (1.1) is

$$
a(t)=a_{0} t^{2 / 3},
$$

where $a_{0}$ is an integration constant. ${ }^{19}$ This model describes an open geometry (the $K=0$ and spatial sections are diffeomorphic to $\mathbb{R}^{3}$ ) in the presence of a constant nonzero energy density distribution. Even though the EdeS space-time is conformally flat, its causal structure is quite different from asymptotically flat geometries. In particular, and unlike Minkowski or Schwarzschild, the past particle horizons exist. The EdeS space-time is a good approximation to the large scale structure of the universe during a matter dominated phase, when the averaged (over space and time) energy density evolves adiabatically and pressures are vanishingly small, as, e.g., immediately after inflation. ${ }^{35}$ This justifies why such a metric is adopted to model the collapse of overdensity perturbations in the early matter dominated phase that followed inflation.

The Einstein and de Sitter model of the universe is the simplest nonempty expanding model with the line element,

$$
d s^{2}=-d t^{2}+a_{0}^{2} t^{4 / 3}\left(d x^{2}+d y^{2}+d z^{2}\right),
$$

in comoving coordinates. ${ }^{11}$ It was first proposed jointly by Einstein and de Sitter (the EdeS model). ${ }^{10}$ The observations of the microwave radiation fit in with this model. ${ }^{9}$ The result of this case also correctly describes the early epoch, even in a universe with curvature different from zero (Ref. 5, Sec. 8.2). Recently it was used in Ref. 29 to study cosmological black holes. The key observation for that approach is that the line element can also be written in the conformally flat form,

$$
d s^{2}=\tau^{4}\left[-d \tau^{2}+d r^{2}+r^{2}\left(d \theta^{2}+\sin ^{2} \theta d \phi^{2}\right)\right],
$$

where the timelike coordinates are related by $d \tau / d t=(3 t)^{-2 / 3}$. The last form is an asymptotic for the Schwarzschild metric whose line element may be written in the form

$$
d s^{2}=\tau^{4}\left[-\left(1-\frac{2 m}{r}\right) d t^{2}+\frac{4 m}{r} d t d r+\left(1+\frac{2 m}{r}\right) d r^{2}+r^{2}\left(d \theta^{2}+\sin ^{2} \theta d \phi^{2}\right)\right],
$$

where $t$ and $r$ are timelike and spacelike coordinates related to the standard Schwarzschild coordinates $\bar{t}$ and $\bar{r}$ by

$$
t=\bar{t}+2 m \ln \left|\frac{\bar{r}}{2 m}-1\right|, \quad r=\bar{r} .
$$

The fact that the resulting metric is asymptotically Einstein and de Sitter with the source reducing to a comoving pure dust at null infinity is used in Ref. 29. In this sense the solution could be interpreted as a black hole in the asymptotic background of the Einstein and de Sitter universe.

The covariant d'Alambert's operator in the Einstein and de Sitter space-time is

$$
\square_{g} \psi=-\left(\frac{\partial}{\partial t}\right)^{2} \psi+t^{-4 / 3} \sum_{i=1,2,3}\left(\frac{\partial}{\partial x^{i}}\right)^{2} \psi-\frac{2}{t} \frac{\partial}{\partial t} \psi
$$

Consequently, the covariant wave equation with the source term $f$ written in the coordinates is 


$$
\left(\frac{\partial}{\partial t}\right)^{2} \psi-t^{-4 / 3} \sum_{i=1,2,3}\left(\frac{\partial}{\partial x^{i}}\right)^{2} \psi+\frac{2}{t} \frac{\partial}{\partial t} \psi=f
$$

The last equation belongs to the family of the non-Fuchsian partial differential equations. There is very advanced theory of such equations (see, e.g., Ref. 18 and references therein). In this note we investigate the initial value problem for this equation and give the representation formulas for the solutions with any dimension $n \in \mathbb{N}$ of the spatial variable $x \in \mathbb{R}^{n}$.

Equation (1.2) is strictly hyperbolic in the domain with $t>0$. On the hypersurface $t=0$ its coefficients have singularities that make the study of the initial value problem difficult. Then, the speed of propagation is equal to $t^{-2 / 3}$ for every $t \in \mathbb{R} \backslash\{0\}$. Equation (1.2) is not Lorentz invariant, which brings additional difficulties.

The classical works on the Tricomi and Gellerstedt equations (see, e.g., Refs. 4, 6, 8, and 31) appeal to the singular Cauchy problem for the Euler-Poisson-Darboux equation,

$$
\Delta u=u_{t t}+\frac{c}{t} u_{t}, \quad c \in \mathbb{C}
$$

and to the Asgeirsson mean value theorem when handling a high-dimensional case. Here $\Delta$ is the Laplace operator on the flat metric, $\Delta:=\sum_{i=1}^{n}\left(\partial^{2} / \partial x_{i}^{2}\right)$.

We use the approach suggested in Ref. 32 and reduce the problem for Eq. (1.2) to the Cauchy problem for the free wave equation in Minkowski space-time: $v_{t t}-\Delta v=0$. To us, this approach seems to be more immediate than the one that uses the Euler-Poisson-Darboux equation. More precisely, in the present note we utilize the solution $v=v(x, t ; b)$ to the Cauchy problem,

$$
\begin{cases}v_{t t}-\Delta v=0, & t>0, \quad x \in \mathbb{R}^{n} \\ v(x, 0)=\varphi(x, b), & v_{t}(x, 0)=0, \quad x \in \mathbb{R}^{n}\end{cases}
$$

with the parameter $b \in B \subseteq \mathbb{R}$. We denote that solution by $v_{\varphi}=v_{\varphi}(x, t ; b)$. In the case of function $\varphi$ independent of parameter, we skip $b$ and simply write $v_{\varphi}=v_{\varphi}(x, t)$. There are well-known explicit representation formulas for the solution of the last problem. We write those formulas to make the present note self-contained. If $n=1$, and $\varphi(x, t)=f(x, t) \in C^{\infty}(\mathbb{R} \times \mathbb{R}), B=\mathbb{R}$, then

$$
v_{f}(x, t ; b)=\frac{1}{2}\{f(x+t, b)+f(x-t, b)\}, \quad t \in \mathbb{R}, \quad x \in \mathbb{R} .
$$

For $f \in C^{\infty}\left(\mathbb{R}^{n} \times \mathbb{R}\right)$ and for odd $n=2 m+1, m \in \mathbb{N}$,

$$
v_{f}(x, t ; b)=\frac{\partial}{\partial t}\left(\frac{1}{t} \frac{\partial}{\partial t}\right)^{(n-3) / 2} \frac{t^{n-2}}{\omega_{n-1} c_{0}^{(n)}} \int_{S^{n-1}} f(x+t y, b) d S_{y}, \quad t \in \mathbb{R}, \quad x \in \mathbb{R}^{n},
$$

where, $c_{0}^{(n)}=1 \cdot 3 \cdot 5 \cdot \ldots \cdot(n-2)$, while for $x \in \mathrm{R}^{n}$ with even $n=2 m, m \in \mathbb{N}$,

$$
v_{f}(x, t ; b)=\frac{\partial}{\partial t}\left(\frac{1}{t} \frac{\partial}{\partial t}\right)^{(n-2) / 2} \frac{2 t^{n-1}}{\omega_{n-1} c_{0}^{(n)}} \int_{B_{1}^{n}(0)} \frac{1}{\sqrt{1-|y|^{2}}} f(x+t y, b) d V_{y}, \quad t \in \mathbb{R}, x \in \mathbb{R}^{n},
$$

where $c_{0}^{(n)}=1 \cdot 3 \cdot 5 \cdot \ldots \cdot(n-1)$. (See, e.g., Theorems 4.1,4.2. $\left.{ }^{24}\right)$ In particular, if $f$ is independent of $t$, then $v_{f}(x, t ; b)$ does not depend on $b$ and we briefly write $v_{f}(x, t)$.

The straightforward application of the formulas obtained in Ref. 32 to the Cauchy problem for Eq. (1.2) decidedly does not work, but it reveals a surprising link to the Einstein and de Sitter space-time. To demonstrate that link we note that the "principal part" of Eq. (1.2) belongs to the family of the Tricomi-type equations (in the case of odd $l$ it is Gellerstedt equation),

$$
u_{t t}-t^{l} \Delta u=0,
$$

where $l \in \mathbb{N}$. According to Ref. 32 the solution to the Cauchy problem, 


$$
u_{t t}-t^{l} \Delta u=f(x, t), \quad u(x, 0)=\varphi_{0}(x), \quad u_{t}(x, 0)=\varphi_{1}(x),
$$

with the smooth functions $f, \varphi_{0}$, and $\varphi_{1}$ can be represented as follows:

$$
\begin{aligned}
u(x, t)= & 2^{2-2 \gamma} \frac{\Gamma(2 \gamma)}{\Gamma^{2}(\gamma)} \int_{0}^{1}\left(1-s^{2}\right)^{\gamma-1} v_{\varphi_{0}}(x, \phi(t) s) d s \\
& +t 2^{2 \gamma} \frac{\Gamma(2-2 \gamma)}{\Gamma^{2}(1-\gamma)} \int_{0}^{1}\left(1-s^{2}\right)^{-\gamma} v_{\varphi_{1}}(x, \phi(t) s) d s \\
& +2 c_{k} \int_{0}^{t} d b \int_{0}^{\phi(t)-\phi(b)} d r E(r, t ; 0, b) v_{f}(x, r ; b), \quad x \in \mathbb{R}^{n}, \quad t>0,
\end{aligned}
$$

with the kernel

$$
E(r, t ; 0, b):=\left((\phi(b)+\phi(t))^{2}-r^{2}\right)^{-\gamma} F\left(\gamma, \gamma ; 1 ; \frac{(\phi(t)-\phi(b))^{2}-r^{2}}{(\phi(t)+\phi(b))^{2}-r^{2}}\right) .
$$

Here $F(\gamma, \gamma ; 1 ; \zeta)$ is the hypergeometric function (see, e.g., Ref. 1), while $k:=l / 2, \phi(t)$ $:=t^{(k+1)} /(k+1), \gamma:=k /(2 k+2)$, and $c_{k}=(k+1)^{-k /(k+1)} 2^{-1 /(k+1)}$. The equation with $l \in \mathbb{N}, x \in \mathbb{R}$, and $f=0$ is studied in Ref. 30 by means of the partial Fourier transform and the confluent hypergeometric function. That approach gives parametrix of the Cauchy problem, and as a consequence, a complete description of the propagation of the $C^{\infty}$-singularities.

Suppose now that we are looking for the simplest possible kernel $E(r, t ; 0, b)(1.9)$ of the last integral transform. In the hierarchy of the hypergeometric functions the simplest one, that is different from the constant, is a linear function. That simplest function $F(a, b ; 1 ; \zeta)$ has the parameters $a=b=-1$ and coincides with $1+\zeta$. The parameter $l$ leading to such function $F(-1$, $-1 ; 1 ; \zeta)=1+\zeta$ is exactly the exponent $l=-4 / 3$ of the wave equation (and of the metric tensor) in the Einstein and de Sitter space-time.

It is evident that the first term of the representation (1.8), as it is written, is meaningless if $\gamma=-1$. This indicates the fact that the Cauchy problem is not well posed anymore for the equation with $l=-4 / 3$. The next theorem also shows how the "lower order term" of Eq. (1.2) affects the Cauchy problem. The main result of this paper is the following theorem.

Theorem 1.1: Assume that $\varphi_{i} \in C^{[n / 2]+3-i}\left(\mathbb{R}^{n}\right), i=0,1, f(x, t) \in C^{[n / 2]+2}\left(\mathbb{R}^{n} \times(0, \infty)\right)$, and that with some $\varepsilon>0$ one has

$$
\left|\partial_{x}^{\alpha} f(x, t)\right|+\left|t \partial_{t} \partial_{x}^{\beta} f(x, t)\right| \leq C_{\alpha} t^{\varepsilon-2} \quad \text { for all } x \in \mathbb{R}^{n}, \quad \text { and for all small } t>0,
$$

and for every $\alpha, \beta,|\alpha| \leq[n / 2]+2,|\beta| \leq[n / 2]+1$. Then the solution $\psi=\psi(x, t)$ to the problem,

$$
\left\{\begin{array}{l}
\psi_{t t}-t^{-4 / 3} \Delta \psi+2 t^{-1} \psi_{t}=f(x, t), \quad t>0, \quad x \in \mathbb{R}^{n} \\
\lim _{t \rightarrow 0} t \psi(x, t)=\varphi_{0}(x), \quad \lim _{t \rightarrow 0}\left(t \psi_{t}(x, t)+\psi(x, t)+3 t^{-1 / 3} \Delta \varphi_{0}(x)\right)=\varphi_{1}(x), \quad x \in \mathbb{R}^{n},
\end{array}\right.
$$

is given by

$$
\begin{aligned}
\psi(x, t)= & \frac{3}{2} t^{2} \int_{0}^{1} d b \int_{0}^{1-b^{1 / 3}} d s b v_{f}\left(x, 3 t^{1 / 3} s ; t b\right)\left(1+b^{2 / 3}-s^{2}\right) \\
& +t^{-1} v_{\varphi_{0}}\left(x, 3 t^{1 / 3}\right)-3 t^{-2 / 3}\left(\partial_{t} v_{\varphi_{0}}\right)\left(x, 3 t^{1 / 3}\right)+\frac{3}{2} \int_{0}^{1} v_{\varphi_{1}}\left(x, 3 t^{1 / 3} s\right)\left(1-s^{2}\right) d s .
\end{aligned}
$$

The theorem shows that one cannot anticipate the well posedness in the Cauchy problem for the wave equation in the Einstein and de Sitter space-time. In fact, it gives a structure of singularity of the solution at the point $t=0$, which hints at the proper initial conditions which have to be 
prescribed for the solution. The initial conditions prescribed in the previous theorem are the Cauchy conditions modified to the so-called weighted initial conditions in order to adjust them to the equation. For the Euler-Poisson-Darboux equation (1.3) one can find such weighted initial conditions, for instance, in Refs. 4 and 25 as well as in the references therein. The existence and uniqueness of the solutions for the initial value problem with the weighted initial conditions for the Euler-Poisson-Darboux equation and for Eq. (1.3) with the time-dependent $c$ are proven in Ref. 7 by application of the Fourier transform in $x$-variable, as well as some transformations which reduce the equation to the confluent hypergeometric equation.

Theorem 1.1 can be used to obtain some important properties for the solutions of the wave equation in Einstein and de Sitter space-time, which are inherited from the solutions of the wave equation in Minkowski space-time. In particular, as a consequence of the previous theorem, in Sec. III, for the initial value problem (1.10) with $n \geq 2, f=0$, and $\varphi_{0}=0$, we obtain the following $L^{p}-L^{q}$ estimate:

$$
\left\|(-\Delta)^{-s} \psi(\cdot, t)\right\|_{L^{q\left(\mathrm{R}^{n}\right)}} \leq C t^{(1 / 3)(2 s-n(1 / p-1 / q))}\left\|\varphi_{1}\right\|_{L^{p}\left(\mathrm{R}^{n}\right)}, \quad t>0,
$$

provided that $s \geq 0,1<p \leq 2,1 / p+1 / q=1, \quad(1 / 2)(n+1)(1 / p-1 / q) \leq 2 s \leq n(1 / p-1 / q)$, and $n(1 / p-1 / q)-1<2 s$. Similar estimates hold for the problem with general $\varphi_{0}$ and $f$. Thus, in the present paper we prepare all necessary tools that will allow us to study in the forthcoming paper the solvability of semilinear wave equation in the Einstein and de Sitter space-time. Having in mind the scale invariance of the equation and also the applications (see, e.g., Refs. 16 and 28), special attention will be given to the self-similar solutions. Results analogous to those presented in this note have already proven to be a good tool in the study of self-similar solutions. ${ }^{35}$

This note is organized as follows. In Sec. II we prove the main theorem and give some of its extensions (Theorems 2.1 and 2.2) that allow stronger singularity in the source term. Section III is devoted to the application of the main theorem, namely, to the derivation of the $L^{p}-L^{q}$ estimates.

The EdeS model recently became a focus of interest for an increasing number of authors. (See, e.g., Refs. 2, 11-15, 22, 23, and 29 and references therein.) We believe that the initial value problem and the explicit representation formulas obtained in the present paper fill the gap in the existing literature on the wave equation in the EdeS space-time.

\section{PROOF OF THE MAIN THEOREM}

If we denote

$$
\mathcal{L}:=\partial_{t}^{2}-t^{-4 / 3} \Delta+2 t^{-1} \partial_{t}, \quad \mathcal{S}:=\partial_{t}^{2}-t^{-4 / 3} \Delta,
$$

then we can easily check for $t \neq 0$ the following operator identity:

$$
t^{-1} \circ \mathcal{S} \circ t=\mathcal{L}
$$

The last equation suggests a change of the unknown function $\psi$ with $u$ such that $\psi=t^{-1} u$. Then the problem for $u$ is as follows:

$$
\left\{\begin{array}{l}
u_{t t}-t^{-4 / 3} \Delta u=g(x, t), \quad t>0, \quad x \in \mathbb{R}^{n} \\
\lim _{t \rightarrow 0} u(x, t)=\varphi_{0}(x), \quad x \in \mathbb{R}^{n} \\
\lim _{t \rightarrow 0}\left(u_{t}(x, t)+3 t^{-1 / 3} \Delta \varphi_{0}(x)\right)=\varphi_{1}(x), \quad x \in \mathbb{R}^{n},
\end{array}\right.
$$

where $g(x, t)=t f(x, t)$. Therefore it is enough to find a representation of the solution of the last problem. We discuss it in three separate cases of: (f) with $\varphi_{0}=\varphi_{1}=0 ;\left(\varphi_{0}\right)$ with $f=0$ and $\varphi_{1}=0$; $\left(\varphi_{1}\right)$ with $f=0$ and $\varphi_{0}=0$. 
We will use the following property of the resolving operator of the problem (1.4): if $P$ $=P\left(D_{x}\right)$ is a pseudodifferential operator and $\tau=\tau(t)$ is a smooth function of time, then the operator $\tau(t) P\left(D_{x}\right)$ "commutes" with the resolving operator of the problem (1.4). More precisely, the following identity can easily be verified:

$$
v_{\tau P\left(D_{x}\right) f}(x, t ; b)=\tau(b) P\left(D_{x}\right) v_{f}(x, t ; b) \text { for all } f \in C^{\infty}\left(\mathbb{R}^{n} \times(0, \infty)\right) .
$$

The operator $\mathcal{S}$ belongs to the family of the Tricomi-type operators,

$$
\mathcal{T}:=\partial_{t}^{2}-t^{l} \Delta
$$

where $l \in \mathbb{R}$. The Cauchy problem for such operators with positive $l$, that is, for the case of multiple characteristics, is well developed (see, e.g., Ref. 33 and references therein). The fundamental solutions of the operator and the representation formulas for the solutions of the Cauchy problem are given in Ref. 32. The results of Ref. 32 allow us to write an ansatz for the solutions of the equation of (2.2). This ansatz has been used in Ref. 17 to extend the range of admissible values of $l$ to negative numbers for the problem with data on the hyperplane $t \neq 0$. Here we use this ansatz to consider the weighted initial value problem (2.2) with data on the plane $t=0$, where coefficients and source term are singular. As we already emphasized, it is interesting that the case of $l=-4 / 3$, that is the case of Einstein and de Sitter space-time, is an exceptional case in the sense that it simplifies the Gauss' hypergeometric function $F(\gamma, \gamma ; 1 ; z)$ appearing in the fundamental solutions constructed in Ref. 32, to the linear function $F(-1,-1 ; 1 ; z)=1+z$.

The case of $(\mathbf{f})$. Assume that $f \in C^{[n / 2]+2}\left(\mathbb{R}^{n} \times(0, \infty)\right)$ and for every given multi-indexes $\alpha, \beta$, $|\alpha| \leq[n / 2]+2,|\beta| \leq[n / 2]+1$, the following inequality holds:

$$
\left|\partial_{x}^{\alpha} f(x, t)\right|+\left|t \partial_{t} \partial_{x}^{\beta} f(x, t)\right| \leq C t^{\varepsilon-2} \quad \text { for all } x \in \mathbb{R}^{n}, \quad t \in(0, T),
$$

for small positive $T$. We have to prove that the solution to the problem,

$$
\left\{\begin{array}{l}
\mathcal{L} \psi=f, \quad t>0, \quad x \in \mathbb{R}^{n} \\
\lim _{t \rightarrow 0} \psi(x, t)=0, \quad \lim _{t \rightarrow 0} \psi_{t}(x, t)=0, \quad x \in \mathbb{R}^{n},
\end{array}\right.
$$

is given by

$$
\psi(x, t)=\frac{3}{2} t^{2} \int_{0}^{1} d b \int_{0}^{1-b^{1 / 3}} d s b v_{f}\left(x, 3 t^{1 / 3} s ; t b\right)\left(1+b^{2 / 3}-s^{2}\right)
$$

Here the function $v_{f}(x, r ; t)$ is given by (1.5)-(1.7), if $n=1, n$ is odd, and $n$ is even, respectively.

It is sufficient to check the properties of the function $u=u(x, t)=t \psi(x, t)$, which solves the equation $\mathcal{S} u=g$ with $g(x, t)=t f(x, t)$. Hence, we can restrict ourselves to the representation,

$$
\begin{aligned}
u(x, t) & =\frac{3}{2} t^{2} \int_{0}^{1} d b \int_{0}^{1-b^{1 / 3}} d s v_{g}(x, \phi(t) s ; t b)\left(1+b^{2 / 3}-s^{2}\right) \\
& =\frac{1}{18} \int_{0}^{t} d l \int_{0}^{\phi(t)-\phi(l)} d r v_{g}(x, r ; l)\left(\phi^{2}(t)+\phi^{2}(l)-r^{2}\right),
\end{aligned}
$$

and take into account the identity $v_{g}(x, r ; b)=b v_{f}(x, r ; b)$. Here $\phi(t):=3 t^{1 / 3}$. First we prove that the integral is convergent and that it represents a $C^{2}\left(\mathbb{R}^{n} \times(0, \infty)\right)$-function. We will skip the subindex $g$ in the remainder of the proof. It is evident that $v(x, r ; b) \in C^{2}\left(\mathbb{R}^{n} \times(0, \infty)\right)$ and that

$$
\left|\partial_{x}^{\alpha} v(x, r ; b)\right|+\left|\partial_{r} v(x, r ; b)\right|+\left|\partial_{r}^{2} v(x, r ; b)\right|+\left|b \partial_{b} v(x, r ; b)\right| \leq C b^{-1+\varepsilon}
$$

if $|\alpha| \leq 2$. It follows 


$$
|v(x, \phi(t) s ; t b)| \leq C t^{-1+\varepsilon} b^{-1+\varepsilon} .
$$

Then we use the last inequality and the first formula of (2.5) in the following inequalities:

$$
|u(x, t)| \leq C t^{1+\varepsilon} \int_{0}^{1} b^{-1+\varepsilon} d b \int_{0}^{1-b^{1 / 3}} d s\left(1+b^{2 / 3}-s^{2}\right) \leq C_{\varepsilon} t^{1+\varepsilon} .
$$

The first formula of (2.5) leads to the estimate for the derivative,

$$
\begin{aligned}
\left|\frac{\partial}{\partial t} u(x, t)\right| \leq & \left|3 t \int_{0}^{1} d b \int_{0}^{1-b^{1 / 3}} d s v(x, \phi(t) s ; t b)\left(1+b^{2 / 3}-s^{2}\right)\right| \\
& +\left|\frac{3}{2} t^{4 / 3} \int_{0}^{1} d b \int_{0}^{1-b^{1 / 3}} d s s\left(\partial_{r} v\right)(x, \phi(t) s ; t b)\left(1+b^{2 / 3}-s^{2}\right)\right| \\
& +\left|\frac{3}{2} t^{2} \int_{0}^{1} d b \int_{0}^{1-b^{1 / 3}} d s b\left(\partial_{b} v\right)(x, \phi(t) s ; t b)\left(1+b^{2 / 3}-s^{2}\right)\right|,
\end{aligned}
$$

that implies

$$
\begin{aligned}
\left|\frac{\partial}{\partial t} u(x, t)\right| \leq & C_{\varepsilon} t^{\varepsilon} \int_{0}^{1} b^{-1+\varepsilon} d b \int_{0}^{1-b^{1 / 3}} d s\left(1+b^{2 / 3}-s^{2}\right) \\
& +C_{\varepsilon} t^{1 / 3+\varepsilon} \int_{0}^{1} b^{-1+\varepsilon} d b \int_{0}^{1-b^{1 / 3}} d s s\left(1+b^{2 / 3}-s^{2}\right) \\
& +C_{\varepsilon} t^{\varepsilon} \int_{0}^{1} b^{-1+\varepsilon} d b \int_{0}^{1-b^{1 / 3}} d s\left(1+b^{2 / 3}-s^{2}\right) .
\end{aligned}
$$

Thus, the estimates (2.6) and (2.7) lead to the initial conditions

$$
\lim _{t \rightarrow 0} u(x, t)=0, \quad \lim _{t \rightarrow 0} u_{t}(x, t)=0 .
$$

It remains to verify the equation. For the derivative $(\partial / \partial t) u(x, t)$ we use the second formula of (2.5) and obtain

$$
\begin{aligned}
\frac{\partial}{\partial t} u(x, t)= & \phi^{\prime}(t) \frac{1}{18} \int_{0}^{t} v(x, \phi(t)-\phi(l) ; l)\left(\phi^{2}(t)+\phi^{2}(l)-(\phi(t)-\phi(l))^{2}\right) d l \\
& +2 \phi^{\prime}(t) \phi(t) \frac{1}{18} \int_{0}^{t} d l \int_{0}^{\phi(t)-\phi(l)} d r v(x, r ; l) \\
= & \frac{1}{18}\left(\phi^{2}(t)\right)^{\prime} \int_{0}^{t} v(x, \phi(t)-\phi(l) ; l) \phi(l) d l \\
& +\frac{1}{18}\left(\phi^{2}(t)\right)^{\prime} \int_{0}^{t} d l \int_{0}^{\phi(t)-\phi(l)} d r v(x, r ; l) .
\end{aligned}
$$

For the second order derivative $\left(\partial^{2} / \partial t^{2}\right) u(x, t)$, since $\frac{1}{18}\left(\phi^{2}(t)\right)^{\prime} v(x, 0 ; t) \phi(t)=g(x, t)$ we derive from the last equation 


$$
\begin{aligned}
\frac{\partial^{2}}{\partial t^{2}} u(x, t)= & g(x, t)+\frac{1}{18}\left(\phi^{2}(t)\right)^{\prime \prime} \int_{0}^{t} v(x, \phi(t)-\phi(l) ; l) \phi(l) d l \\
& +\frac{1}{18}\left(\phi^{2}(t)\right)^{\prime} \phi^{\prime}(t) \int_{0}^{t} v_{r}(x, \phi(t)-\phi(l) ; l) \phi(l) d l+\frac{1}{18}\left(\phi^{2}(t)\right)^{\prime \prime} \int_{0}^{t} d l \int_{0}^{\phi(t)-\phi(l)} d r v(x, r ; l) \\
& +\frac{1}{18}\left(\phi^{2}(t)\right)^{\prime} \phi^{\prime}(t) \int_{0}^{t} v(x, \phi(t)-\phi(l) ; l) d l
\end{aligned}
$$

By means of the second formula of (2.5) and equation of (1.4) for the function $\Delta u$ we derive

$$
\Delta u(x, t)=\frac{1}{18} \int_{0}^{t} d l \int_{0}^{\phi(t)-\phi(l)} d r \partial_{r}^{2} v(x, r ; l)\left(\phi^{2}(t)+\phi^{2}(l)-r^{2}\right) .
$$

It follows

$$
\begin{aligned}
\Delta u(x, t)= & \frac{1}{9} \phi(t) \int_{0}^{t} \partial_{r} v(x, \phi(t)-\phi(l) ; l) \phi(l) d l-\frac{1}{18} \int_{0}^{t} \partial_{r} v(x, 0 ; l)\left(\phi^{2}(t)+\phi^{2}(l)\right) d l \\
& +\frac{1}{9} \int_{0}^{t} d l \int_{0}^{\phi(t)-\phi(l)} d r r \partial_{r} v(x, r ; l) .
\end{aligned}
$$

Since $\partial_{r} v(x, 0 ; l)=0$, one more integration by parts yields

$$
\begin{aligned}
\Delta u(x, t)= & \frac{1}{9} \phi(t) \int_{0}^{t} \partial_{r} v(x, \phi(t)-\phi(l) ; l) \phi(l) d l+\frac{1}{9} \int_{0}^{t} v(x, \phi(t)-\phi(l) ; l)(\phi(t)-\phi(l)) d l \\
& -\frac{1}{9} \int_{0}^{t} d l \int_{0}^{\phi(t)-\phi(l)} d r v(x, r ; l) .
\end{aligned}
$$

Hence according to (2.9) and (2.10) the application of the operator $\partial_{t}^{2}-t^{-4 / 3} \Delta$ to the function $u$ $=u(x, t)$ gives

$$
\begin{aligned}
u_{t t}(x, t)-t^{-4 / 3} \Delta u(x, t)= & g(x, t)+\frac{1}{18}\left(\phi^{2}(t)\right)^{\prime \prime} \int_{0}^{t} v(x, \phi(t)-\phi(l) ; l) \phi(l) d l \\
& +\frac{1}{18}\left(\phi^{2}(t)\right)^{\prime} \phi^{\prime}(t) \int_{0}^{t} v_{r}(x, \phi(t)-\phi(l) ; l) \phi(l) d l \\
& +\frac{1}{18}\left(\phi^{2}(t)\right)^{\prime} \phi^{\prime}(t) \int_{0}^{t} v(x, \phi(t)-\phi(l) ; l) d l \\
& -t^{-4 / 3}\left\{\frac{1}{9} \phi(t) \int_{0}^{t} v_{r}(x, \phi(t)-\phi(l) ; l) \phi(l) d l\right. \\
& \left.+\frac{1}{9} \int_{0}^{t} v(x, \phi(t)-\phi(l) ; l)(\phi(t)-\phi(l)) d l\right\} \\
= & g(x, t)+\frac{1}{18}\left(\phi^{2}(t)\right)^{\prime \prime} \int_{0}^{t} v(x, \phi(t)-\phi(l) ; l) \phi(l) d l \\
& +\frac{1}{18}\left(\phi^{2}(t)\right)^{\prime} \phi^{\prime}(t) \int_{0}^{t} v(x, \phi(t)-\phi(l) ; l) d l
\end{aligned}
$$




$$
\begin{aligned}
& -t^{-4 / 3} \frac{1}{9} \int_{0}^{t} v(x, \phi(t)-\phi(l) ; l)(\phi(t)-\phi(l)) d l \\
= & g(x, t)+\frac{1}{18}\left(\phi^{2}(t)\right)^{\prime} \phi^{\prime}(t) \int_{0}^{t} v(x, \phi(t)-\phi(l) ; l) d l \\
& -t^{-4 / 3} \frac{1}{9} \phi(t) \int_{0}^{t} v(x, \phi(t)-\phi(l) ; l) d l \\
= & g(x, t) .
\end{aligned}
$$

Thus, for this case the theorem is proven.

One can allow more strong singularity of the source function $f$ at $t=0$. More precisely, one can reduce the case with such singularity to the one of Theorem 1.1 if the initial condition is modified. That is done in the next theorem.

Theorem 2.1: Assume that $f(x, t) \in C^{[n / 2]+4}\left(\mathbb{R}^{n} \times(0, \infty)\right), t^{2} f(x, t) \in C\left(\mathbb{R}^{n} \times[0, \infty)\right)$ and that

$$
\left|\partial_{x}^{\alpha} f(x, t)\right|+\left|t \partial_{t} \partial_{x}^{\beta} f(x, t)\right| \leq C_{\alpha} t^{-2} \quad \text { for all } t \in(0, T), \quad x \in \mathbb{R}^{n},
$$

and for every $\alpha, \beta,|\alpha| \leq[n / 2]+4,|\beta| \leq[n / 2]+3$. Denote $f_{0}(x):=\lim _{t \rightarrow 0} t^{2} f(x, t)$ and suppose that with some $\varepsilon>0$ for the functions $f(x, t)$ and $f_{0}(x) \in C^{[n / 2]+4}\left(\mathbb{R}^{n}\right)$ the following inequality is fulfilled:

$$
\left|\partial_{x}^{\alpha}\left(t f(x, t)-t^{-1} f_{0}(x)\right)\right|+\left|t \partial_{t} \partial_{x}^{\beta}\left(t f(x, t)-t^{-1} f_{0}(x)\right)\right| \leq C_{\alpha} t^{\varepsilon-1} \quad \text { for all } t \in(0, T), \quad x \in \mathbb{R}^{n},
$$

and for every $\alpha, \beta,|\alpha| \leq[n / 2]+2,|\beta| \leq[n / 2]+1$.

Then the solution $\psi=\psi(x, t)$ of the problem,

$$
\left\{\begin{array}{l}
\psi_{t t}-t^{-4 / 3} \Delta \psi+2 t^{-1} \psi_{t}=f(x, t), \quad t>0, \quad x \in \mathbb{R}^{n} \\
\lim _{t \rightarrow 0}(t \psi(x, t))=0, \quad \lim _{t \rightarrow 0}\left(t \psi_{t}(x, t)+\psi(x, t)-f_{0}(x) \ln t\right)=0, \quad x \in \mathbb{R}^{n},
\end{array}\right.
$$

is given by

$$
\begin{aligned}
\psi(x, t)= & \frac{1}{t} f_{0}(x) \tau(t)+\frac{1}{18 t} \int_{0}^{t} d l \int_{0}^{\phi(t)-\phi(l)} d r\left(\phi^{2}(t)+\phi^{2}(l)-r^{2}\right) \\
& \times\left(l v_{f}(x, r ; l)-l^{-1} v_{f_{0}}(x, r)+l^{-4 / 3} \tau(l) \Delta v_{f_{0}}(x, r)\right),
\end{aligned}
$$

where $\tau(t):=\int_{0}^{t} \ln s d s$.

Proof: Consider the new unknown function $w(x, t):=u-f_{0}(x) \tau(t)$. Then

$$
\mathcal{S} w(x, t)=t f(x, t)-\mathcal{S}\left(f_{0}(x) \tau(t)\right)=t f(x, t)-\left(t^{-1} f_{0}(x)-t^{-4 / 3} \tau(t) \Delta f_{0}(x)\right)=h(x, t),
$$

where we have denoted

$$
h(x, t):=t\left(f(x, t)-t^{-2} f_{0}(x)\right)+t^{-4 / 3} \tau(t) \Delta f_{0}(x) .
$$

According to the condition of the theorem with some $\varepsilon>0$ we have

$$
\left|\partial_{x}^{\alpha} h(x, t)\right|+\left|t \partial_{t} \partial_{x}^{\beta} h(x, t)\right| \leq C_{\alpha} t^{\varepsilon-1} \quad \text { for all } t \in(0, T), x \in \mathbb{R}^{n},
$$

$\alpha, \beta,|\alpha| \leq[n / 2]+2,|\beta| \leq[n / 2]+1$, that allows us to write representation (2.5) for the solution $w=w(x, t)$, 


$$
w(x, t)=\frac{1}{18} \int_{0}^{t} d l \int_{0}^{\phi(t)-\phi(l)} d r v_{h}(x, r ; l)\left(\phi^{2}(t)+\phi^{2}(l)-r^{2}\right) .
$$

On the other hand, according to Theorem 1.1, the function $w=w(x, t)$ satisfies initial conditions

$$
\lim _{t \rightarrow 0} w(x, t)=0, \quad \lim _{t \rightarrow 0} w_{t}(x, t)=0 .
$$

Consequently,

$$
\begin{aligned}
& \lim _{t \rightarrow 0} u(x, t)=\lim _{t \rightarrow 0}\left(w(x, t)+f_{0}(x) \tau(t)\right)=0, \\
& \lim _{t \rightarrow 0}\left(u_{t}(x, t)-f_{0}(x) \ln t\right)=\lim _{t \rightarrow 0} w_{t}(x, t)=0 .
\end{aligned}
$$

For the function $\psi=\psi(x, t)=t^{-1} u(x, t)$ this implies the initial conditions of (2.11). To prove representation formula (2.12), we note that

$$
\begin{aligned}
v_{h}(x, r ; b) & =v_{t\left(f(x, t)-t^{-2} f_{0}(x)\right)+t^{-4 / 3} \tau(t) \Delta f_{0}(x)}(x, r ; b) \\
& =v_{t f(x, t)}(x, r ; b)-v_{t^{-1} f_{0}(x)}(x, r ; b)+v_{t^{-4 / 3}} \tau(t) \Delta f_{0}(x) \\
& =b v_{f}(x, r ; b)-b^{-1} v_{f_{0}}(x, r ; b)+b^{-4 / 3} \tau(b) \Delta v_{f_{0}}(x, r ; b) \\
& =b v_{f}(x, r ; b)-b^{-1} v_{f_{0}}(x, r)+b^{-4 / 3} \tau(b) \Delta v_{f_{0}}(x, r) .
\end{aligned}
$$

Then we use (2.13) to write

$$
w(x, t)=\frac{1}{18} \int_{0}^{t} d l \int_{0}^{\phi(t)-\phi(l)} d r\left(\phi^{2}(t)+\phi^{2}(l)-r^{2}\right)\left(l v_{f}(x, r ; l)-l^{-1} v_{f_{0}}(x, r)+l^{-4 / 3} \tau(l) \Delta v_{f_{0}}(x, r)\right) .
$$

Thus, the representation

$$
\begin{aligned}
u(x, t)= & f_{0}(x) \tau(t)+\frac{1}{18} \int_{0}^{t} d l \int_{0}^{\phi(t)-\phi(l)} d r\left(\phi^{2}(t)+\phi^{2}(l)-r^{2}\right) \\
& \times\left(l v_{f}(x, r ; l)-l^{-1} v_{f_{0}}(x, r)+l^{-4 / 3} \tau(l) \Delta v_{f_{0}}(x, r)\right)
\end{aligned}
$$

implies (2.12). Theorem is proven.

The last theorem does not exhaust the possible singularities of the source terms. The next theorem gives behavior of the solution as $t \rightarrow 0$ if the source term is more singular.

Theorem 2.2: Assume that $f(x, t) \in C^{[n / 2]+4}\left(\mathbb{R}^{n} \times(0, \infty)\right)$ and that with number $a \in(2,8 / 3)$ one has $t^{a} f(x, t) \in C\left(\mathbb{R}^{n} \times[0, \infty)\right)$ and

$$
\left|\partial_{x}^{\alpha} f(x, t)\right|+\left|t \partial_{t} \partial_{x}^{\beta} f(x, t)\right| \leq C_{\alpha} t^{-a} \quad \text { for all } t \in(0, T), \quad x \in \mathbb{R}^{n},
$$

and for every $\alpha, \beta,|\alpha| \leq[n / 2]+4,|\beta| \leq[n / 2]+1$. Denote $f_{0}(x):=\lim _{t \rightarrow 0} t^{a} f(x, t)$ and suppose that with some $\varepsilon>0$ for the functions $f=f(x, t)$ and $f_{0}=f_{0}(x) \in C^{[n / 2]+4}\left(\mathbb{R}^{n}\right)$ the following inequality is fulfilled:

$$
\left|\partial_{x}^{\alpha}\left(t f(x, t)-t^{1-a} f_{0}(x)\right)\right|+\left|t \partial_{t} \partial_{x}^{\beta}\left(t f(x, t)-t^{1-a} f_{0}(x)\right)\right| \leq C_{\alpha} t^{\varepsilon-1} \quad \text { for all } t \in(0, T), \quad x \in \mathbb{R}^{n},
$$

and for every $\alpha, \beta,|\alpha| \leq[n / 2]+2,|\beta| \leq[n / 2]+1$. Denote $\tau(t):=(3-a)^{-1}(2-a)^{-1} t^{3-a}$.

Then the solution $\psi=\psi(x, t)$ of the problem, 


$$
\left\{\begin{array}{l}
\psi_{t t}-t^{-4 / 3} \Delta \psi+2 t^{-1} \psi_{t}=f(x, t), \quad t>0, \quad x \in \mathbb{R}^{n} \\
\lim _{t \rightarrow 0}\left(t \psi(x, t)-\frac{1}{(3-a)(2-a)} t^{3-a} f_{0}(x)\right)=0, \quad x \in \mathbb{R}^{n} \\
\lim _{t \rightarrow 0}\left(t \psi_{t}(x, t)+\psi(x, t)-\frac{1}{2-a} t^{2-a} f_{0}(x)\right)=0, \quad x \in \mathbb{R}^{n},
\end{array}\right.
$$

is given by

$$
\begin{aligned}
\psi(x, t)= & \frac{1}{t} f_{0}(x) \tau(t)+\frac{1}{18 t} \int_{0}^{t} d l \int_{0}^{\phi(t)-\phi(l)} d r\left(\phi^{2}(t)+\phi^{2}(l)-r^{2}\right) \\
& \times\left(l v_{f}(x, r ; l)-l^{1-a} v_{f_{0}}(x, r)+l^{-4 / 3} \tau(l) \Delta v_{f_{0}}(x, r)\right) .
\end{aligned}
$$

Proof: Consider the new unknown function $w(x, t):=u-f_{0}(x) \tau(t)$. Then

$$
\mathcal{S} w(x, t)=t f(x, t)-\mathcal{S}\left(f_{0}(x) \tau(t)\right)=t f(x, t)-\left(t^{1-a} f_{0}(x)-t^{-4 / 3} \tau(t) \Delta f_{0}(x)\right)=h(x, t),
$$

where we have denoted

$$
h(x, t):=t\left(f(x, t)-t^{-a} f_{0}(x)\right)+t^{-4 / 3} \tau(t) \Delta f_{0}(x) .
$$

According to the condition of the theorem with some $\varepsilon>0$ we have

$$
\left|\partial_{x}^{\alpha} h(x, t)\right|+\left|t \partial_{x}^{\beta} h_{t}(x, t)\right| \leq C_{\alpha} t^{\varepsilon-1} \quad \text { for all } t \in(0, T), \quad x \in \mathbb{R}^{n},
$$

$\alpha, \beta,|\alpha| \leq[n / 2]+2,|\beta| \leq[n / 2]+1$, that allows us to write representation (2.13). On the other hand, according to Theorem 1.1, the function $w=w(x, t)$ satisfies initial conditions (2.14). Consequently,

$$
\begin{gathered}
\lim _{t \rightarrow 0}\left(u(x, t)-f_{0}(x) \tau(t)\right)=\lim _{t \rightarrow 0} w(x, t)=0, \\
\lim _{t \rightarrow 0}\left(u_{t}(x, t)-f_{0}(x) \tau^{\prime}(t)\right)=\lim _{t \rightarrow 0} w_{t}(x, t)=0 .
\end{gathered}
$$

For the function $\psi=\psi(x, t)=t^{-1} u(x, t)$ this implies the initial conditions,

$$
\lim _{t \rightarrow 0}\left(t \psi(x, t)-f_{0}(x) \tau(t)\right)=0, \quad \lim _{t \rightarrow 0}\left(t \psi_{t}(x, t)+\psi(x, t)-f_{0}(x) \tau^{\prime}(t)\right)=0,
$$

which coincide with ones of (2.15). To prove representation formula (2.16), we note that

$$
\begin{aligned}
v_{h}(x, r ; b) & =v_{t\left(f(x, t)-t^{-a} f_{0}(x)\right)+t^{-4 / 3} \tau(t) \Delta f_{0}(x)}(x, r ; b) \\
& =v_{t f(x, t)}(x, r ; b)-v_{t^{1-a} f_{0}(x)}(x, r ; b)+v_{t^{-4 / 3}} \tau(t) \Delta f_{0}(x)(x, r ; b) \\
& =b v_{f}(x, r ; b)-b^{1-a} v_{f_{0}}(x, r)+b^{-4 / 3} \tau(b) \Delta v_{f_{0}}(x, r) .
\end{aligned}
$$

Then

$$
\begin{aligned}
w(x, t)= & \frac{1}{18} \int_{0}^{t} d l \int_{0}^{\phi(t)-\phi(l)} d r v_{h}(x, r ; l)\left(\phi^{2}(t)+\phi^{2}(l)-r^{2}\right) \\
= & \frac{1}{18} \int_{0}^{t} d l \int_{0}^{\phi(t)-\phi(l)} d r\left(\phi^{2}(t)+\phi^{2}(l)-r^{2}\right) \\
& \times\left(l v_{f}(x, r ; l)-l^{1-a} v_{f_{0}}(x, r)+l^{-4 / 3} \tau(l) \Delta v_{f_{0}}(x, r)\right) .
\end{aligned}
$$

Thus, the following representation 


$$
\begin{aligned}
u(x, t)= & f_{0}(x) \tau(t)+\frac{1}{18} \int_{0}^{t} d l \int_{0}^{\phi(t)-\phi(l)} d r\left(\phi^{2}(t)+\phi^{2}(l)-r^{2}\right) \\
& \times\left(l v_{f}(x, r ; l)-l^{1-a} v_{f_{0}}(x, r)+l^{-4 / 3} \tau(l) \Delta v_{f_{0}}(x, r)\right)
\end{aligned}
$$

for the function $u=u(x, t)$ implies (2.16). Theorem is proven.

The case of $\left(\varphi_{0}\right)$. In this case $f=0$ and $\varphi_{1}=0$. One can find in the literature different approaches for the construction of the solutions of the Fuchsian and non-Fuchsian partial differential equations. (See, e.g., Refs. 18 and 20.) The next two lemmas give for $f=0$ behavior of the solutions of the equation of (1.10) near the point of singularity $t=0$ of the coefficients.

Lemma 2.3: For $\varphi_{0} \in C_{0}^{[n / 2]+3}\left(\mathbb{R}^{n}\right)$ the function

$$
u(x, t)=v_{\varphi_{0}}\left(x, 3 t^{1 / 3}\right)-3 t^{1 / 3}\left(\partial_{r} v_{\varphi_{0}}\right)\left(x, 3 t^{1 / 3}\right)
$$

solves the problem

$$
\begin{cases}\mathcal{S} u=0, \quad x \in \mathbb{R}^{n}, & t>0 \\ \lim _{t \rightarrow 0} u(x, t)=\varphi_{0}(x), & \lim _{t \rightarrow 0}\left(u_{t}(x, t)+3 t^{-1 / 3} \Delta \varphi_{0}(x)\right)=0, \quad x \in \mathbb{R}^{n}\end{cases}
$$

Here $v_{\varphi}\left(x, 3 t^{1 / 3}\right)$ is the value of the solution $v(x, r)$ to the Cauchy problem for the wave equation, $v_{r r}-\Delta v=0, v(x, 0)=\varphi(x), v_{t}(x, 0)=0$, taken at the point $(x, r)=\left(x, 3 t^{1 / 3}\right)$.

Proof: We verify it by straightforward calculations. It is evident that

$$
\Delta u(x, t)=\Delta v_{\varphi_{0}}\left(x, 3 t^{1 / 3}\right)-3 t^{1 / 3}\left(\partial_{r} \Delta v_{\varphi_{0}}(x, r)\right)_{r=3 t^{1 / 3}}
$$

Denote

$$
v_{0}(x, t)=v_{\varphi_{0}}\left(x, 3 t^{1 / 3}\right), \quad v_{1}(x, t)=-3 t^{1 / 3}\left(\partial_{r} v_{\varphi_{0}}(x, r)\right)_{r=3 t^{1 / 3}} .
$$

Then, for the derivatives $\partial_{t} v_{0}(x, t)$ and $\partial_{t}^{2} v_{0}(x, t)$ we have

$$
\begin{gathered}
\partial_{t} v_{0}(x, t)=t^{-2 / 3}\left(\partial_{r} v_{\varphi_{0}}(x, r)\right)_{r=3 t^{1 / 3}} \\
\partial_{t}^{2} v_{0}(x, t)=-\frac{2}{3} t^{-5 / 3}\left(\partial_{r} v_{\varphi_{0}}(x, r)\right)_{r=3 t^{1 / 3}+t^{-4 / 3}}\left(\partial_{r}^{2} v_{\varphi_{0}}(x, r)\right)_{r=3 t^{1 / 3}}
\end{gathered}
$$

At the mean time for the derivatives $\partial_{t} v_{1}(x, t)$ and $\partial_{t}^{2} v_{1}(x, t)$ we have

$$
\begin{gathered}
\partial_{t} v_{1}(x, t)=-t^{-2 / 3}\left(\partial_{r} v_{\varphi_{0}}(x, r)\right)_{r=3 t^{1 / 3}-3 t^{-1 / 3}\left(\partial_{r}^{2} v_{\varphi_{0}}(x, r)\right)_{r=3 t^{1 / 3}},} \\
\partial_{t}^{2} v_{1}(x, t)=\frac{2}{3} t^{-5 / 3}\left(\partial_{r} v_{\varphi_{0}}(x, r)\right)_{r=3 t^{1 / 3}}-3 t^{-1}\left(\partial_{r}^{3} v_{\varphi_{0}}(x, r)\right)_{r=3 t^{1 / 3}} .
\end{gathered}
$$

Hence, for the first order derivative $\partial_{t} u(x, t)$ and for the second order derivative $\partial_{t}^{2} u(x, t)$ we have

$$
\begin{gathered}
\partial_{t} u(x, t)=-3 t^{-1 / 3}\left(\partial_{r}^{2} v_{\varphi_{0}}(x, r)\right)_{r=3 t^{1 / 3},} \\
\partial_{t}^{2} u(x, t)=t^{-4 / 3}\left(\partial_{r}^{2} v_{\varphi_{0}}(x, r)\right)_{r=3 t^{1 / 3}-3 t^{-1}\left(\partial_{r}^{3} v_{\varphi_{0}}(x, r)\right)_{r=3 t^{1 / 3},}}
\end{gathered}
$$

respectively. Consequently, using (2.18) and (2.19), and the definition of $v_{\varphi}$ we obtain 


$$
\begin{aligned}
\partial_{t}^{2} u(x, t)-t^{-4 / 3} \Delta u(x, t)= & t^{-4 / 3}\left(\partial_{r}^{2} v_{\varphi_{0}}(x, r)\right)_{r=3 t^{1 / 3}}-3 t^{-1}\left(\partial_{r}^{3} v_{\varphi_{0}}(x, r)\right)_{r=3 t^{1 / 3}}-t^{-4 / 3}\left(\Delta v_{\varphi_{0}}(x, r)\right)_{r=3 t^{1 / 3}} \\
& +3 t^{-1}\left(\partial_{r} \Delta v_{\varphi_{0}}(x, r)\right)_{r=3 t^{1 / 3}} \\
= & t^{-4 / 3}\left(\partial_{r}^{2} v_{\varphi_{0}}(x, r)-\Delta v_{\varphi_{0}}(x, r)\right)_{r=3 t^{1 / 3}}-3 t^{-1}\left(\partial_{r}\left(\partial_{r}^{2} v_{\varphi_{0}}(x, r)-\Delta v_{\varphi_{0}}(x, r)\right)\right)_{r=3 t^{1 / 3}} \\
= & 0 .
\end{aligned}
$$

Thus, the function $u=u(x, t)$ solves the equation $u_{t t}(x, t)-t^{-4 / 3} \Delta u(x, t)=0$. Lemma is proven.

Corollary 2.4: The function $\psi=t^{-1} u(x, t)$ solves the problem $(1.10)$ with $\varphi_{1}=0$ and with $f=0$, that is,

$$
\left\{\begin{array}{l}
\psi_{t t}(x, t)-t^{-4 / 3} \Delta \psi(x, t)+2 t^{-1} \psi_{t}(x, t)=0 \\
\lim _{t \rightarrow 0} t \psi(x, t)=\varphi_{0}, \quad \lim _{t \rightarrow 0}\left(t \psi_{t}(x, t)+\psi(x, t)+3 t^{-1 / 3} \Delta \varphi_{0}(x)\right)=0, \quad x \in \mathbb{R}^{n}
\end{array}\right.
$$

In particular, the corollary shows that for the given dimension $n \in \mathbb{N}$ Huygens' principle is valid for some particular waves propagating in the Einstein and de Sitter model of the universe if and only if it is valid for the waves propagating in Minkowski space-time (cf. with Refs. 26, 32, and 36).

The case of $\left(\varphi_{1}\right)$. In this case $f=0$ and $\varphi_{0}=0$.

Lemma 2.5: For $\varphi_{1} \in C_{0}^{[n / 2]+2}\left(\mathbb{R}^{n}\right)$ the function

$$
u(x, t)=t \frac{3}{2} \int_{0}^{1} v_{\varphi_{1}}(x, \phi(t) s)\left(1-s^{2}\right) d s, \quad x \in \mathbb{R}^{n}, \quad t>0,
$$

solves the problem

$$
\left\{\begin{array}{l}
\mathcal{S} u=0, \quad x \in \mathbb{R}^{n}, \quad t>0 \\
\lim _{t \rightarrow 0} u(x, t)=0, \quad \lim _{t \rightarrow 0} u_{t}(x, t)=\varphi_{1}(x), \quad x \in \mathbb{R}^{n}
\end{array}\right.
$$

Here $v_{\varphi}(x, \phi(t) s)$ is the value of the solution $v(x, r)$ to the Cauchy problem for the wave equation, $v_{r r}-\Delta v=0, v(x, 0)=\varphi(x), v_{t}(x, 0)=0$, taken at the point $(x, r)=(x, \phi(t) s)$, while $\phi(t)=3 t^{1 / 3}$.

Proof: We prove the lemma by straightforward calculations. We have

$$
u(x, t)=t \frac{3}{2} \int_{0}^{1} v_{\varphi_{1}}(x, \phi(t) s)\left(1-s^{2}\right) d s=\frac{1}{18} \int_{0}^{\phi(t)} v_{\varphi_{1}}(x, r)\left(\phi^{2}(t)-r^{2}\right) d r .
$$

For the first order derivative we derive

$$
\partial_{t} u(x, t)=\partial_{t} \frac{1}{18} \int_{0}^{\phi(t)} v_{\varphi_{1}}(x, r)\left(\phi^{2}(t)-r^{2}\right) d r=\frac{1}{3} t^{-1 / 3} \int_{0}^{\phi(t)} v_{\varphi_{1}}(x, r) d r
$$

while for the second order derivative using the last equation and integration by parts, we obtain

$$
\begin{aligned}
\partial_{t}^{2} u(x, t) & =-\frac{1}{9} t^{-4 / 3} \int_{0}^{\phi(t)} v_{\varphi_{1}}(x, r) d r+\frac{1}{3} t^{-1} v_{\varphi_{1}}(x, \phi(t)) \\
& =-\frac{1}{9} t^{-4 / 3}\left(\left.v_{\varphi_{1}}(x, r) r\right|_{0} ^{\phi(t)}-\int_{0}^{\phi(t)} r\left(\partial_{r} v_{\varphi_{1}}\right)(x, r) d r\right)+\frac{1}{3} t^{-1} v_{\varphi_{1}}(x, \phi(t)) \\
& =-\frac{1}{9} t^{-4 / 3}\left(v_{\varphi_{1}}(x, \phi(t)) \phi(t)-\int_{0}^{\phi(t)} r\left(\partial_{r} v_{\varphi_{1}}\right)(x, r) d r\right)+\frac{1}{3} t^{-1} v_{\varphi_{1}}(x, \phi(t)) .
\end{aligned}
$$

Consequently, 


$$
\partial_{t}^{2} u(x, t)=\frac{1}{9} t^{-4 / 3} \int_{0}^{\phi(t)} r\left(\partial_{r} v_{\varphi_{1}}\right)(x, r) d r
$$

At the same time, we have

$$
\Delta u(x, t)=\frac{1}{18} \int_{0}^{\phi(t)} \Delta v_{\varphi_{1}}(x, r)\left(\phi^{2}(t)-r^{2}\right) d r .
$$

Then Eqs. (2.21) and (2.22) imply

$$
\begin{aligned}
u_{t t}(x, t)-t^{-4 / 3} \Delta u(x, t)= & \frac{1}{9} t^{-4 / 3}\left(\left.\frac{r^{2}}{2}\left(\partial_{r} v_{\varphi_{1}}\right)(x, r)\right|_{0} ^{\phi(t)}-\int_{0}^{\phi(t)} \frac{r^{2}}{2}\left(\partial_{r}^{2} v_{\varphi_{1}}\right)(x, r) d r\right) \\
& -t^{-4 / 3} \frac{1}{18} \int_{0}^{\phi(t)} \Delta v_{\varphi_{1}}(x, r)\left(\phi^{2}(t)-r^{2}\right) d r \\
= & \frac{1}{9} t^{-4 / 3}\left(\frac{\phi^{2}(t)}{2}\left(\partial_{r} v_{\varphi_{1}}\right)(x, \phi(t))-\int_{0}^{\phi(t)} \frac{r^{2}}{2}\left(\partial_{r}^{2} v_{\varphi_{1}}\right)(x, r) d r\right) \\
& -t^{-4 / 3} \frac{1}{18} \int_{0}^{\phi(t)} \Delta v_{\varphi_{1}}(x, r)\left(\phi^{2}(t)-r^{2}\right) d r \\
= & \frac{1}{18} t^{-4 / 3} \phi^{2}(t)\left(\partial_{r} v_{\varphi_{1}}\right)(x, \phi(t))-\frac{1}{18} t^{-4 / 3} \int_{0}^{\phi(t)} r^{2}\left(\partial_{r}^{2} v_{\varphi_{1}}\right)(x, r) d r \\
& -t^{-4 / 3} \frac{1}{18} \int_{0}^{\phi(t)} \Delta v_{\varphi_{1}}(x, r)\left(\phi^{2}(t)-r^{2}\right) d r \\
= & \frac{1}{2} t^{-2 / 3}\left(\partial_{r} v_{\varphi_{1}}\right)(x, \phi(t))-\frac{1}{2} t^{-2 / 3} \int_{0}^{\phi(t)} \Delta v_{\varphi_{1}}(x, r) d r .
\end{aligned}
$$

The definition of the function $v_{\varphi_{1}}$ suggests that the function $u=u(x, t)$ solves the equation

$$
u_{t t}(x, t)-t^{-4 / 3} \Delta u(x, t)=\frac{1}{2} t^{-2 / 3}\left\{\left(\partial_{r} v_{\varphi_{1}}\right)(x, \phi(t))-\int_{0}^{\phi(t)}\left(\partial_{r}^{2} v_{\varphi_{1}}\right)(x, r) d r\right\}=0 .
$$

Finally, we verify the second initial condition by means of the l'Hospital's rule,

$$
\lim _{t \rightarrow 0} u_{t}(x, t)=\lim _{t \rightarrow 0} \frac{1}{3} t^{-1 / 3} \int_{0}^{\phi(t)} v_{\varphi_{1}}(x, r) d r=\lim _{t \rightarrow 0} v_{\varphi_{1}}(x, \phi(t))=v_{\varphi_{1}}(x, 0)=\varphi_{1}(x) .
$$

Lemma is proven.

Corollary 2.6: The function $\psi=t^{-1} u(x, t)$ solves the problem (1.10) with $\varphi_{0}=0$ and without source term $f$, that is,

$$
\left\{\begin{array}{l}
\psi_{t t}-t^{-4 / 3} \Delta \psi+2 t^{-1} \psi_{t}=0, \quad t>0, \quad x \in \mathbb{R}^{n} \\
\lim _{t \rightarrow 0} t \psi(x, t)=0, \quad \lim _{t \rightarrow 0}\left(t \psi_{t}(x, t)+\psi(x, t)\right)=\varphi_{1}(x), \quad x \in \mathbb{R}^{n} .
\end{array}\right.
$$

The last corollary completes the proof of Theorem 1.1 .

In particular, Corollary 2.4 and Corollary 2.6 show that, because of the integration in the formula (2.20), for all $n \in \mathbb{N}$ Huygens' principle is not valid for waves propagating in the Einstein and de Sitter model of the universe, unless $\varphi_{1}=0$ and $f=0$. 


\section{III. $L^{p}-L^{q}$ ESTIMATES}

The representation formula (1.11) of Theorem 1.1 can be used to reproduce for the solutions of the wave equation in Einstein and de Sitter space-time some important properties which possess the solutions of the wave equation in Minkowski space-time. Among them there are estimates of the norm of solution in various functional spaces, such as $L^{p}$, Sobolev spaces, Besov spaces, and others. These estimates provide a useful tool to prove local and global in time existence theorems. ${ }^{24,27,34,35}$

In this short note we derive such estimates in the Lebesgue spaces only. First we remind these estimates. If $n \geq 2$, then for the solution $v=v(x, t)$ of the Cauchy problem for the wave equation in Minkowski space-time

$$
v_{t t}-\Delta v=0, \quad v(x, 0)=\varphi(x), \quad v_{t}(x, 0)=0,
$$

with $\varphi(x) \in C_{0}^{\infty}\left(\mathbb{R}^{n}\right)$ one has (see, e.g., Refs. 3 and 21) the following so-called $L^{p}-L^{q}$ decay estimate:

$$
\left\|(-\Delta)^{-s} v(\cdot, t)\right\|_{L^{q\left(\mathrm{R}^{n}\right)}} \leq C t^{2 s-n(1 / p-1 / q)}\left\|_{\varphi}\right\|_{L^{p}\left(\mathbb{R}^{n}\right)} \text { for all } t>0,
$$

provided that $s \geq 0,1<p \leq 2,1 / p+1 / q=1$, and $(1 / 2)(n+1)(1 / p-1 / q) \leq 2 s \leq n(1 / p-1 / q)$.

Then, for the solution $v=v(x, t)$ of the Cauchy problem for the wave equation,

$$
v_{t t}-\Delta v=0, \quad v(x, 0)=0, \quad v_{t}(x, 0)=\varphi(x),
$$

there is the $L^{p}-L^{q}$ estimate

$$
\left\|(-\Delta)^{-s} v(\cdot, t)\right\|_{L^{q}\left(\mathbb{R}^{n}\right)} \leq C t^{2 s+1-n(1 / p-1 / q)}\left\|_{\varphi}\right\|_{L^{p}\left(\mathrm{R}^{n}\right)} \quad \text { for all } t>0,
$$

under the conditions $s \geq 0,1<p \leq 2,1 / p+1 / q=1$, and $(1 / 2)(n+1)(1 / p-1 / q)-1 \leq 2 s \leq n(1 / p$ $-1 / q)$.

The case of $\left(\varphi_{0}\right)$. According to Theorem 1.1, for the problem with $\varphi_{1}=0$ and $f=0$ the function $\psi=\psi(x, t)$ can be represented as follows:

$$
\psi(x, t)=t^{-1} v_{\varphi_{0}}\left(x, 3 t^{1 / 3}\right)-3 t^{-2 / 3}\left(\partial_{t} v_{\varphi_{0}}\right)\left(x, 3 t^{1 / 3}\right) .
$$

Here for $\varphi_{0} \in C_{0}^{\infty}\left(\mathbb{R}^{n}\right)$ the function $v_{\varphi_{0}}\left(x, 3 t^{1 / 3}\right)$ coincides with the value $v\left(x, 3 t^{1 / 3}\right)$ of the solution $v(x, t)$ of the Cauchy problem (3.1). Hence for $s \geq 0$ by means of application of (3.2) we obtain

$$
\left\|(-\Delta)^{-s} v_{\varphi_{0}}\left(\cdot, 3 t^{1 / 3}\right)\right\|_{L^{q}\left(\mathbb{R}^{n}\right)} \leq C t^{(1 / 3)(2 s-n(1 / p-1 / q))}\left\|\varphi_{0}\right\|_{L^{p}\left(\mathbb{R}^{n}\right)}, \quad t>0 .
$$

To estimate the second term of (3.5) we apply (3.4) with $s \geq 0$,

$$
\left\|(-\Delta)^{-s}\left(\partial_{r} v_{\varphi_{0}}\right)\left(\cdot, 3 t^{1 / 3}\right)\right\|_{L^{q}\left(\mathbb{R}^{n}\right)} \leq C t^{(1 / 3)(2 s+1-n(1 / p-1 / q))}\left\|\Delta \varphi_{0}\right\|_{L^{p}\left(\mathbb{R}^{n}\right)}, \quad t>0,
$$

provided that $(1 / 2)(n+1)(1 / p-1 / q)-1 \leq 2 s \leq n(1 / p-1 / q)$. Consequently, if $s \geq 0,1<p \leq 2$, $1 / p+1 / q=1$, and $(1 / 2)(n+1)(1 / p-1 / q) \leq 2 s \leq n(1 / p-1 / q)$, then for the problem with $\varphi_{1}=0$ and $f=0$ we obtain

$$
\begin{aligned}
\left\|(-\Delta)^{-s} \psi(\cdot, t)\right\|_{L^{q\left(\mathbb{R}^{n}\right)}} & \leq C t^{-1+(1 / 3)(2 s-n(1 / p-1 / q))}\left\|\varphi_{0}\right\|_{L^{p}\left(\mathbb{R}^{n}\right)}+C t^{-2 / 3} t^{(1 / 3)(2 s+1-n(1 / p-1 / q))}\left\|\Delta \varphi_{0}\right\|_{L^{p}\left(\mathbb{R}^{n}\right)} \\
& \leq C t^{(1 / 3)(2 s-n(1 / p-1 / q))}\left(t^{-1}\left\|\varphi_{0}\right\|_{L^{p}\left(\mathbb{R}^{n}\right)}+t^{-1 / 3}\left\|\Delta \varphi_{0}\right\|_{L^{p}\left(\mathbb{R}^{n}\right)}\right), \quad t>0 .
\end{aligned}
$$

Thus, we have proven the following proposition.

Proposition 3.1: Suppose that $s \geq 0,1<p \leq 2,1 / p+1 / q=1$, and $(1 / 2)(n+1)(1 / p-1 / q)$ $\leq 2 s \leq n(1 / p-1 / q)$. Then the solution $\psi=\psi(x, t)$ to the problem

$$
\left\{\begin{array}{l}
\psi_{t t}-t^{-4 / 3} \Delta \psi+2 t^{-1} \psi_{t}=0, \quad t>0, \quad x \in \mathbb{R}^{n} \\
\lim _{t \rightarrow 0} t \psi(x, t)=\varphi_{0}(x), \quad \lim _{t \rightarrow 0}\left(t \psi_{t}(x, t)+\psi(x, t)+3 t^{-1 / 3} \Delta \varphi_{0}(x)\right)=0, \quad x \in \mathbb{R}^{n},
\end{array}\right.
$$


with $\varphi_{0} \in C_{0}^{\infty}\left(\mathbb{R}^{n}\right)$ satisfies the following estimate:

$$
\left\|(-\Delta)^{-s} \psi(\cdot, t)\right\|_{L^{q\left(\mathbb{R}^{n}\right)}} \leq C t^{(1 / 3)(2 s-1-n(1 / p-1 / q))}\left(t^{-2 / 3}\left\|\varphi_{0}\right\|_{L^{p}\left(\mathbb{R}^{n}\right)}+\left\|\Delta \varphi_{0}\right\|_{L^{p}\left(\mathbb{R}^{n}\right)}\right), \quad t>0,
$$

with the constant $C$ independent of $\varphi_{0}$.

The case of $\left(\varphi_{1}\right)$. For the problem with $\varphi_{0}=0$ and $f=0$ the function $\psi=\psi(x, t)$ due to Theorem 1.1 can be represented as follows:

$$
\psi(x, t)=\frac{3}{2} \int_{0}^{1} v_{\varphi_{1}}(x, \phi(t) s)\left(1-s^{2}\right) d s, \quad x \in \mathbb{R}^{n}, \quad t>0 .
$$

Then we obtain for $s, n, p$, and $q$ such that $2 s-n(1 / p-1 / q)>-1$, the following estimate:

$$
\begin{aligned}
\left\|(-\Delta)^{-s} \psi(\cdot, t)\right\|_{L^{q\left(\mathbb{R}^{n}\right)}} & \leq \frac{3}{2} \int_{0}^{1}\left\|(-\Delta)^{-s} v_{\varphi_{1}}(\cdot, \phi(t) s)\right\|_{L^{q\left(\mathbb{R}^{n}\right)}}\left(1-s^{2}\right) d s \\
& \leq C \int_{0}^{1} t^{(1 / 3)(2 s-n(1 / p-1 / q))} s^{(2 s-n(1 / p-1 / q))}\left\|\varphi_{1}\right\|_{L^{p}\left(\mathbb{R}^{n}\right)}\left(1-s^{2}\right) d s \\
& \leq C t^{(1 / 3)(2 s-n(1 / p-1 / q))}\left\|\varphi_{1}\right\|_{L^{p}\left(\mathbb{R}^{n}\right)} \int_{0}^{1} s^{2 s-n(1 / p-1 / q)}\left(1-s^{2}\right) d s .
\end{aligned}
$$

Thus, in this case we have proven the following proposition.

Proposition 3.2: Suppose that $s \geq 0,1<p \leq 2,1 / p+1 / q=1,2 s-n(1 / p-1 / q)>-1$, and $(1 / 2)(n+1)(1 / p-1 / q) \leq 2 s \leq n(1 / p-1 / q)$. Then the solution $\psi=\psi(x, t)$ to the problem

$$
\left\{\begin{array}{l}
\psi_{t t}-t^{-4 / 3} \Delta \psi+2 t^{-1} \psi_{t}=0, \quad t>0, \quad x \in \mathbb{R}^{n} \\
\lim _{t \rightarrow 0} t \psi(x, t)=0, \quad \lim _{t \rightarrow 0}\left(t \psi_{t}(x, t)+\psi(x, t)\right)=\varphi_{1}(x), \quad x \in \mathbb{R}^{n},
\end{array}\right.
$$

with $\varphi_{1} \in C_{0}^{\infty}\left(\mathbb{R}^{n}\right)$ satisfies the following estimate:

$$
\left\|(-\Delta)^{-s} \psi(\cdot, t)\right\|_{L^{q}\left(\mathrm{R}^{n}\right)} \leq C t^{(1 / 3)(2 s-n(1 / p-1 / q))}\left\|\varphi_{1}\right\|_{L^{p}\left(\mathrm{R}^{n}\right)}, \quad t>0,
$$

with the constant $C$ independent of $\varphi_{1}$.

The case of (f). According to Theorem 1.1, for the problem with $\varphi_{0}=0$ and $\varphi_{1}=0$ the function $\psi=\psi(x, t)$ can be represented as follows:

$$
\psi(x, t)=\frac{3}{2} t^{2} \int_{0}^{1} d b \int_{0}^{1-b^{1 / 3}} d \tau b v_{f}\left(x, 3 t^{1 / 3} \tau ; t b\right)\left(1+b^{2 / 3}-\tau^{2}\right) .
$$

Consequently, for the problem with $\varphi_{0}=0, \varphi_{1}=0$, and the function $f$ satisfying conditions of the theorem, by application of (3.2) we obtain

$$
\begin{aligned}
\left\|(-\Delta)^{-s} \psi(\cdot, t)\right\|_{L^{q}\left(\mathrm{R}^{n}\right)} & \leq \frac{3}{2} t^{2} \int_{0}^{1} d b \int_{0}^{1-b^{1 / 3}} d \tau b\left\|(-\Delta)^{-s} v_{f}\left(\cdot, 3 t^{1 / 3} \tau ; t b\right)\right\|_{L^{q}\left(\mathbb{R}^{n}\right)}\left(1+b^{2 / 3}-\tau^{2}\right) \\
& \leq C t^{2} \int_{0}^{1} d b \int_{0}^{1-b^{1 / 3}} d \tau b t^{(1 / 3)(2 s-n(1 / p-1 / q))} \tau^{2 s-n(1 / p-1 / q)}\|f(\cdot, t b)\|_{L^{p}\left(\mathbb{R}^{n}\right)}\left(1+b^{2 / 3}-\tau^{2}\right) .
\end{aligned}
$$

For $a=2 s-n(1 / p-1 / q)>-1$ one has

$$
\int_{0}^{1-b^{1 / 3}} \tau^{a}\left(1+b^{2 / 3}-\tau^{2}\right) d \tau=\frac{2}{(a+1)(a+3)}\left(1-b^{1 / 3}\right)^{a+1}\left(1+(a+1) b^{1 / 3}+b^{2 / 3}\right) .
$$

Hence, 


$$
\begin{aligned}
& \left\|(-\Delta)^{-s} \psi(\cdot, t)\right\|_{L^{q\left(\mathrm{R}^{n}\right)}} \\
& \quad \leq C t^{2+(1 / 3)(2 s-n(1 / p-1 / q))} \int_{0}^{1} b\|f(\cdot, t b)\|_{L^{p}\left(\mathrm{R}^{n}\right)} d b \int_{0}^{1-b^{1 / 3}} \tau^{2 s-n(1 / p-1 / q)}\left(1+b^{2 / 3}-\tau^{2}\right) d \tau \\
& \quad \leq C_{n, p, q, s} t^{2+(1 / 3)(2 s-n(1 / p-1 / q))} \int_{0}^{1} b\|f(\cdot, t b)\|_{L^{p}\left(\mathrm{R}^{n}\right)}\left(1-b^{1 / 3}\right)^{a+1}\left(1+(a+1) b^{1 / 3}+b^{2 / 3}\right) d b \\
& \leq C_{n, p, q, s} t^{2+(1 / 3)(2 s-n(1 / p-1 / q))} \int_{0}^{1} b\|f(\cdot, t b)\|_{L^{p\left(\mathrm{R}^{n}\right)}}\left(1-b^{1 / 3}\right)^{2 s-n(1 / p-1 / q)+1} d b .
\end{aligned}
$$

Thus, in this case we have proven the following proposition.

Proposition 3.3: Suppose that $s \geq 0,1<p \leq 2,1 / p+1 / q=1,2 s-n(1 / p-1 / q)>-1$, and $(1 / 2)(n+1)(1 / p-1 / q) \leq 2 s \leq n(1 / p-1 / q)$, and that the function $f$ satisfies conditions of Theorem 1.1. Then for the solution $\psi=\psi(x, t)$ to the problem,

$$
\left\{\begin{array}{l}
\psi_{t t}-t^{-4 / 3} \Delta \psi+2 t^{-1} \psi_{t}=f(x, t), \quad t>0, \quad x \in \mathbb{R}^{n} \\
\lim _{t \rightarrow 0} t \psi(x, t)=0, \quad \lim _{t \rightarrow 0}\left(t \psi_{t}(x, t)+\psi(x, t)\right)=0, \quad x \in \mathbb{R}^{n},
\end{array}\right.
$$

the following estimate:

$$
\left\|(-\Delta)^{-s} \psi(\cdot, t)\right\|_{L^{q\left(\mathbb{R}^{n}\right)}} \leq C_{n, p, q, s} t^{(1 / 3)(2 s-n(1 / p-1 / q))} \int_{0}^{t} \tau\|f(\cdot, \tau)\|_{L^{p}\left(\mathrm{R}^{n}\right)} d \tau
$$

holds with the constant $C_{n, p, q, s}$ independent of $f$.

\section{ACKNOWLEDGMENTS}

This work was initiated during the first and third authors visit Institute of Mathematics of the University of Tsukuba in June 2008. The first and the third authors would like to express their gratitude to the University of Tsukuba for the financial support. They are especially grateful to Professor Kajitani, Professor Wakabayashi, and Professor Isozaki for their hospitality. Finally, the authors thank the referee for useful comments.

\footnotetext{
${ }^{1}$ Bateman, H. and Erdelyi, A., Higher Transcendental Functions (McGraw-Hill, New York, 1954), Vols. 1 and 2.

${ }^{2}$ Blanchard, A., "Cosmological parameters: where are we?" Astrophys. Space Sci. 290, 135 (2004).

${ }^{3}$ Brenner, P., "On $L^{p}-L^{q}$, estimates for the wave-equation," Math. Z. 145, 251 (1975).

${ }^{4}$ Carroll, R. W. and Showalter, R. E., Singular and Degenerate Cauchy Problems. Mathematics in Science and Engineering (Academic, New York, 1976), Vol. 127.

${ }^{5}$ Cheng, T.-P., Relativity, Gravitation And Cosmology: A Basic Introduction (Oxford University Press, Oxford, NY, 2005).

${ }^{6}$ Delache, S. and Leray, J., "Calcul de la solution élémentaire de l'opérateur d'Euler-Poisson-Darboux et de l'opérateur de Tricomi-Clairaut, hyperbolique, d'ordre 2," Bull. Soc. Math. France 99, 313 (1971).

${ }^{7}$ Del Santo, D., Kinoshita, T., and Reissig, M., "Klein-Gordon type equations with a singular time-dependent potential," Rend. Istit. Mat. Univ. Trieste 39, 141 (2007).

${ }^{8}$ Diaz, J. B. and Weinberger, H. F., "A solution of the singular initial value problem for the Euler-Poisson-Darboux equation," Proc. Am. Math. Soc. 4, 703 (1953).

${ }^{9}$ Dirac, P. A. M., "The large numbers hypothesis and the Einstein theory of gravitation," Proc. R. Soc. London, Ser. A $\mathbf{3 6 5}$, 19 (1979).

${ }^{10}$ Einstein, A. and de Sitter, W., "On the relation between the expansion and the mean density of the universe," Proc. Natl. Acad. Sci. U.S.A. 18, 213 (1932).

${ }^{11}$ Ellis, G. and van Elst, H., NATO Advanced Study Institute on Theoretical and Observational Cosmology, Cargèse, France, NATO Science Series, Series C, Mathematical and Physical Sciences, Vol. 541 (Kluwer Academic, Boston, 1999).

${ }^{12}$ Goncalves, S. M. C. V., "Black hole formation from massive scalar field collapse in the Einstein-de Sitter universe," Phys. Rev. D 62, 124006 (2000).

${ }^{13}$ Gron, O. and Hervik, S., Einstein's General Theory of Relativity: With Modern Applications in Cosmology (Springer, New York, 2007).

${ }^{14}$ Hawking, S. W. and Ellis, G. F. R., The Large Scale Structure of Space-Time, Cambridge Monographs on Mathematical Physics, No. 1 (Cambridge University Press, London, 1973).
} 
${ }^{15}$ Hawley, J. F. and Holcomb, K. A., Foundations of Modern Cosmology (Cambridge University Press, New York, 1997).

${ }^{16}$ Henriksen, R. N. and Wesson, P. S., "Self-similar space-times," Astrophys. Space Sci. 53, 429 (1978).

${ }^{17}$ Kinoshita, T. and Yagdjian, K., "On the Cauchy problem for wave equations with time-dependent coefficients," Int. J. Appl. Math. Stat. 13, 1 (2008).

${ }^{18}$ Mandai, T., "Characteristic Cauchy problems for some non-Fuchsian partial differential operators," J. Math. Soc. Jpn. 45, $511(1993)$

${ }^{19}$ Ohanian, H. and Ruffini, R., Gravitation and Spacetime (Norton, New York, 1994).

${ }^{20}$ Parenti, C. and Tahara, H., "Asymptotic expansions of distribution solutions of some Fuchsian hyperbolic equations," Publ. Res. Inst. Math. Sci. 23, 909 (1987).

${ }^{21}$ Pecher, H., " $L^{p}$-abschätzungen und klassische Lösungen für nichtlineare Wellengleichungen.I," Math. Z. 150, 159 (1976).

${ }^{22}$ Peebles, P. J. E., Principles of Physical Cosmology (Princeton University Press, Princeton, NJ, 1993).

${ }^{23}$ Rendall, A. D., Partial Differential Equations in General Relativity, Oxford Graduate Texts in Mathematics No. 16 (Oxford University Press, Oxford, 2008)

${ }^{24}$ Shatah, J. and Struwe, M., Geometric Wave Equations, Courant Lecture Notes in Mathematics No. 2 (American Mathematical Society, Providence, RI, 1998)

${ }^{25}$ Smirnov, M. M., Equations of Mixed Type, Translations of Mathematical Monographs No. 51 (American Mathematical Society, Providence, R.I., 1978) (Translated from the Russian).

${ }^{26}$ Sonego, S. and Faraoni, V., "Huygens' principle and characteristic propagation property for waves in curved spacetimes," J. Math. Phys. 33, 625 (1992).

${ }^{27}$ Strauss, W., CBMS Regional Conference Series in Mathematics (American Mathematical Society, Providence, RI, 1989), Vol. 73 .

${ }^{28}$ Suginohara, T., Taruya, A., and Suto, Y., "Quasi-self-similar evolution of the two-point correlation function: Strongly nonlinear regime in $\Omega_{0}<1$ universes," Astrophys. J. 566, 1 (2002).

${ }^{29}$ Sultana, J. and Dyer, C. C., "Cosmological black holes: A black hole in the Einstein-de Sitter universe," Gen. Relativ. Gravit. 37, 1347 (2005).

${ }^{30}$ Taniguchi, K. and Tozaki, Y., "A hyperbolic equation with double characteristics which has a solution with branching singularities," Math. Japonica 25, 279 (1980).

${ }^{31}$ Weinstein, A., "The singular solutions and the Cauchy problem for generalized Tricomi equations," Commun. Pure Appl. Math. 7, 105 (1954).

${ }^{32}$ Yagdjian, K., "A note on the fundamental solution for the Tricomi-type equation in the hyperbolic domain," J. Differ. Equations 206, 227 (2004).

${ }^{33}$ Yagdjian, K., The Cauchy Problem for Hyperbolic Operators. Multiple Characteristics. Micro-Local Approach (Akademie Verlag, Berlin, 1997).

${ }^{34}$ Yagdjian, K., "Global existence for the $n$-dimensional semilinear Tricomi-type equations," Commun. Partial Differ. Equ. 31, 907 (2006).

${ }^{35}$ Yagdjian, K., "Self-similar solutions of semilinear wave equation with variable speed of propagation," J. Math. Anal. Appl. 336, 1259 (2007).

${ }^{36}$ Yagdjian, K. and Galstian, A., "Fundamental solutions for the Klein-Gordon equation in de Sitter space-time," Commun. Math. Phys. 285, 293 (2009). 